TRANSACTIONS OF THE

AMERICAN MATHEMATICAL SOCIETY

Volume 355, Number 1, Pages 169-176

S 0002-9947(02)03119-7

Article electronically published on September 11, 2002

\title{
BIHARMONIC LIFTS BY MEANS OF PSEUDO-RIEMANNIAN SUBMERSIONS IN DIMENSION THREE
}

\author{
MIGUEL A. JAVALOYES VICTORIA AND MIGUEL A. MEROÑO BAYO
}

\begin{abstract}
We study the total lifts of curves by means of a submersion $\pi$ : $M_{s}^{3} \rightarrow B_{r}^{2}$ that satisfy the condition $\Delta H=\lambda H$ analyzing, in particular, the cases in which the submersion has totally geodesic fibres or integrable horizontal distribution. We also consider in detail the case $\lambda=0$ (biharmonic lifts). Moreover, we obtain a biharmonic lift in $\mathbb{R}^{3}$ by means of a Riemannian submersion that has non-constant mean curvature, getting so a counterexample to the Chen conjecture for $\mathbb{R}^{3}$ with a non-flat Riemannian metric.
\end{abstract}

\section{INTRODUCTION}

Let $M_{s}^{3}$ be a pseudo-Riemannian manifold of dimension 3 and index $s=0,1$, and let $B_{r}^{2}$ be a pseudo-Riemannian surface of index $r=0,1$. Let us consider a pseudo-Riemannian submersion $\pi: M_{s}^{3} \rightarrow B_{r}^{2}$ (see [9] and 10] for more details). Then, given a non-null regular curve $\beta: I \rightarrow B_{r}^{2}$, an interesting question is to relate the geometry of the curve $\beta$ with the geometry of its total lift $P=\pi^{-1}(\beta)$, which is an immersed surface in $M_{s}^{3}$. This relation will depend on the shape of the submersion $\pi$. From this statement it is possible to derive different problems and applications by considering that there are three variable elements: the manifold $M_{s}^{3}$, the surface $B_{r}^{2}$ and the submersion $\pi$. For example, we can fix a concrete submersion in order to reduce the problem of finding surfaces $P$ of $M_{s}^{3}$ satisfying a particular geometric condition to seek curves in $B_{r}^{2}$ satisfying the corresponding condition. This idea was previously used by Pinkall ([11]) to obtain, by means of the Hopf fibration, an infinite family of compact Willmore tori embedded in $\mathbb{S}^{3}$. In 3 the authors used this method to find soliton solutions of the Betchov-Da Rios equation in the anti-De Sitter space. In the same way, this idea also has been used to characterize biharmonic Hopf cylinders, i.e., cylinders with harmonic mean curvature vector field and Hopf cylinders with Jacobi mean curvature vector field (see [4] and [8]).

The problem of finding biharmonic submanifolds has a special importance because of a conjecture of B. Y. Chen, which is stated as follows: the only biharmonic Euclidean submanifolds are the minimal ones (see [6]). This conjecture is true for surfaces in Euclidean space, as Chen proved in 1985. It has also been shown

Received by the editors March 12, 2002 and, in revised form, June 7, 2002.

2000 Mathematics Subject Classification. Primary 53C42; Secondary 53C50.

Key words and phrases. Pseudo-Riemannian submersions, biharmonic surfaces, Chen conjecture.

This research has been partially supported by DGI Grant BFM2001-2871 (MCYT).

The first author was supported by a FPU Predoctoral Grant (MECD). 
for some particular cases of submanifolds (spherical submanifolds, submanifolds of finite type and pseudo-umbilical submanifolds of dimension different from four). However, the conjecture is false if the ambient is, for example, a pseudo-Euclidean space of dimension greater than three (see [7]).

The biharmonicity condition for submanifolds is a particular case of the condition $\Delta H=\lambda H$, where $\Delta$ is the Laplace-Beltrami operator, $H$ is the mean curvature vector field of the immersion and $\lambda$ is a real constant. This equation has been studied in different cases. For instance, all surfaces satisfying the above Laplacian condition have been classified in the constant curvature spaces of dimension three (see [1]). It is interesting to notice that the obtained surfaces have constant mean curvature in every case.

In the present work, we study the mentioned condition in the framework established at the beginning of this introduction. We are going to study the total lift $P$ of a curve $\beta$ in terms of the parameters describing the geometry of the horizontal lifts of $\beta$ (horizontal curves that project on $\beta$ ), since the total lift of $\beta$ can be seen as the disjoint union of its horizontal lifts. Those parameters are none other than the functions curvature and torsion. Actually, these functions determine differentiable functions all over the lift $P$.

Thus, we want to transform the condition $\Delta H=\lambda H$ in terms of the curvature and torsion of the horizontal lifts and the O'Neill invariants $T$ and $A$ associated to the submersion. In this way, we arrive at Theorem 3.1, which states that if the submersion has totally geodesic fibres, then the total lifts $P$ of a curve $\beta$ satisfying the condition $\Delta H=\lambda H$ are those whose horizontal lifts of $\beta$ have constant curvature and torsion, i.e., they are helices.

On the other hand, when we consider submersions with integrable horizontal distribution, we show that the mean curvature function of the total lifts verifying the mentioned condition must be constant on each fibre, but not all over the total lift, which we justify with a counterexample. Moreover, we can choose an example where the total space is an open subset of $\mathbb{R}^{3}$ in such a way that $\lambda$ is null. In particular, the total lift is biharmonic but not minimal. This fact means that the Chen conjecture is not true for $\mathbb{R}^{3}$ with a non-flat Riemannian metric.

It is interesting to compare this fact with the results obtained in [2] for the conjecture of Lawson, which states that the only torus minimally embedded in $\mathbb{S}^{3}$ is the Clifford torus. The authors find a class of non-standard metrics for $\mathbb{S}^{3}$ such that there exist non-congruent flat embedded minimal tori.

We finish the work given a characterization of the total lifts of a curve with constant mean curvature satisfying the condition $\Delta H=\lambda H$ for submersions with integrable horizontal distribution (Theorem [3.6). As a consequence, we obtain that the biharmonic lifts having constant mean curvature by means of a pseudoRiemannian submersion with integrable horizontal distribution are the minimal ones.

We are indebted to the referee for the suggestions given to improve the paper.

\section{Setup}

Let $M_{s}^{3}$ be a pseudo-Riemannian manifold of dimension 3 and index $s=0,1$ and $B_{r}^{2}$ a pseudo-Riemannian surface of index $r=0,1$, and let us consider a pseudoRiemannian submersion $\pi: M_{s}^{3} \rightarrow B_{r}^{2}$. As usual, we will denote the metrics of $M_{s}^{3}$ and $B_{r}^{2}$ by $\langle$,$\rangle , indistinctly, and the induced Riemannian connection by \nabla$. It 
is not difficult to show that if $\beta: I \rightarrow B_{r}^{2}$ is a non-null regular curve, which we assume to be arclength parametrized, then its total lift $P=\pi^{-1}\left(\{\beta(t)\}_{t \in I}\right)$ is a pseudo-Riemannian surface of $M_{s}^{3}$ that is the disjoint union of all horizontal curves that are projected on $\beta$ (horizontal lifts of $\beta$ ).

We are going to do the study of the geometry of $P$ in terms of the parameters describing the geometry of the horizontal lifts of $\beta$. These are precisely the curvature and the torsion of these curves. To this end, let $\left\{X=\beta^{\prime}, N\right\}$ be a Frenet frame along the curve $\beta$ with curvature function $k$. The Frenet equations are written as

$$
\begin{aligned}
& \nabla_{X} X=\varepsilon_{2} k N, \\
& \nabla_{X} N=-\varepsilon_{1} k X,
\end{aligned}
$$

where $\varepsilon_{1}=\langle X, X\rangle, \varepsilon_{2}=\langle N, N\rangle$.

Now, if $\bar{\beta}: I \rightarrow M_{s}^{3}$ is a horizontal lift of $\beta$, let $\bar{X}$ and $\bar{N}$ be the horizontal lifts of $X$ and $N$, respectively. Both $\bar{X}$ and $\bar{N}$ are vector fields along $\bar{\beta}$ and define, by considering all the horizontal lifts of $\beta$, two vector fields on the surface $P$ that are orthonormal since submersions preserve scalar products of horizontal vectors. Furthermore, since $\bar{X}$ is the velocity of the horizontal lifts of $\beta$, it is a vector field tangent to $P$.

Let $V$ be a unit vector field tangent to the fibres, hence tangent to $P$ and orthogonal to $\bar{X}$. Then the triple $\{\bar{X}, \bar{N}, V\}$ is an orthonormal frame of $M_{s}^{3}$ adapted to $P$ that, restricted to any horizontal lift $\bar{\beta}$, is a Frenet frame for $\bar{\beta}$ with curvature function $\bar{k}=\left\langle\nabla_{\bar{X}} \bar{X}, \bar{N}\right\rangle$ and torsion function $\tau=\left\langle\nabla_{\bar{X}} V, \bar{N}\right\rangle$. Actually, it is easy to show that

$$
\left\{\begin{array}{l}
\nabla \bar{X} \bar{X}=\varepsilon_{2} \bar{k} \bar{N}, \\
\nabla \bar{X} \bar{N}=-\varepsilon_{1} \bar{k} \bar{X}-\varepsilon_{3} \tau V, \\
\nabla \bar{X} V=\varepsilon_{2} \tau \bar{N},
\end{array}\right.
$$

where $\varepsilon_{1}=\langle\bar{X}, \bar{X}\rangle, \varepsilon_{2}=\langle\bar{N}, \bar{N}\rangle$ and $\varepsilon_{3}=\langle V, V\rangle$.

Using again that submersions preserve scalar products of horizontal vectors, we can conclude that $k_{\circ} \pi=\bar{k}$ and the torsion is written as $\tau=\left\langle A_{\bar{X}} V, \bar{N}\right\rangle$, where $A$ is the O'Neill invariant associated to the submersion $\pi$.

For the sake of simplicity, we will drop out overbars of the lifted geometrical objects.

As a first step, we are going to find an equivalent equation to the condition $\Delta H=\lambda H$ in terms of the curvature $k$ of the curve $\beta$ and the O'Neill invariants $T$ and $A$ associated to the submersion. For this purpose, we are going to calculate the mean curvature vector field $H$ associated to $P \subset M_{s}^{3}$. This vector field is given by

$$
H=\alpha N,
$$

where $\alpha=\frac{\varepsilon_{2}}{2} \operatorname{Tr}(S)$ is the mean curvature function associated to the immersion, $\operatorname{Tr}$ stands for the trace operator, and $S$ is the shape operator associated to $N$. Since $\{X, V\}$ is an orthonormal frame for the surface $P$, we have

$$
\operatorname{Tr}(S)=\varepsilon_{1}\langle S(X), X\rangle+\varepsilon_{3}\langle S(V), V\rangle .
$$

Since $S(E)=-\nabla_{E} N$, where $E$ is any vector field tangent to $P$, and $[V, X]$ is vertical (see [10] for details), we obtain that

$$
\left\{\begin{array}{l}
S(X)=\varepsilon_{1} k X+\varepsilon_{3} \tau V \\
S(V)=\varepsilon_{1} \tau X-T_{V} N
\end{array}\right.
$$


which leads to

$$
\alpha=\frac{\varepsilon_{2}}{2}\left(\varepsilon_{1} k+\varepsilon_{3}\left\langle T_{V} V, N\right\rangle\right)
$$

We wish to point out that, in particular, if $\pi$ is a totally geodesic submersion (i.e., it has totally geodesic fibres, which is equivalent to $T=0$ ), then $P=\pi^{-1}(\beta)$ is a surface with constant mean curvature if and only if $\beta$ has constant curvature. Moreover, $P$ is a minimal surface in $M_{s}^{3}$ if and only if $\beta$ is a geodesic of $B_{r}^{2}$.

Next we are going to look for a suitable expression for $\Delta H$, where $\Delta$ is the Laplace-Beltrami operator. This operator is expressed in the frame $\{X, V\}$ of $P$ as

$$
\Delta H=-\varepsilon_{1}\left(\nabla_{X} \nabla_{X} H-\nabla_{\bar{\nabla}_{X} X} H\right)-\varepsilon_{3}\left(\nabla_{V} \nabla_{V} H-\nabla_{\bar{\nabla}_{V} V} H\right),
$$

where $\bar{\nabla}$ is the induced connection on $P$.

The proof of the following expression for $\Delta H$ is a straightforward computation (see [5] for details):

$$
\Delta H=2 S(\nabla \alpha)+\alpha \operatorname{Tr}(\bar{\nabla} S)+\left\{\Delta \alpha+\varepsilon_{2} \alpha \operatorname{Tr}\left(S^{2}\right)\right\} N,
$$

where $\nabla \alpha$ stands for the gradient of $\alpha$.

The gradient of the mean curvature function is written as

$$
\nabla \alpha=\varepsilon_{1} X(\alpha) X+\varepsilon_{3} V(\alpha) V
$$

hence, by 2.2), we obtain

$$
\begin{aligned}
S(\nabla \alpha)= & \left(k X(\alpha)+\varepsilon_{1} \varepsilon_{3} V(\alpha) \tau\right) X \\
& +\left(\left\langle T_{V} V, N\right\rangle V(\alpha)+\varepsilon_{1} \varepsilon_{3} X(\alpha) \tau\right) V .
\end{aligned}
$$

On the other hand, from (2.1) the Laplacian of $\alpha$ is expressed as

$$
\Delta \alpha=-\varepsilon_{1} X^{2}(\alpha)-\varepsilon_{3} V^{2}(\alpha)+\varepsilon_{1} \varepsilon_{3}\left\langle T_{V} V, X\right\rangle X(\alpha) .
$$

For the trace of $S^{2}$ we have that

$$
\operatorname{Tr}\left(S^{2}\right)=\varepsilon_{1}\left\langle S^{2}(X), X\right\rangle+\varepsilon_{3}\left\langle S^{2}(V), V\right\rangle,
$$

which, by applying (2.2) again, gives

$$
\operatorname{Tr}\left(S^{2}\right)=k^{2}+2 \varepsilon_{1} \varepsilon_{3} \tau^{2}+\left\langle T_{V} V, N\right\rangle^{2} .
$$

Finally, $\operatorname{Tr}(\bar{\nabla} S)$ is defined by

$$
\operatorname{Tr}(\bar{\nabla} S)=\varepsilon_{1}\left(\bar{\nabla}_{X} S\right)(X)+\varepsilon_{3}\left(\bar{\nabla}_{V} S\right)(V) .
$$

So from (2.1) and (2.2) we obtain, by a straightforward computation, that

$$
\begin{aligned}
\operatorname{Tr}(\bar{\nabla} S)=(X(k)+ & \left.\varepsilon_{1}\left\langle T_{V} V, N\right\rangle\left\langle T_{V} V, X\right\rangle+\varepsilon_{1} \varepsilon_{3} V(\tau)-\varepsilon_{3} k\left\langle T_{V} V, X\right\rangle\right) X \\
& +\left(\varepsilon_{1} \varepsilon_{3} X(\tau)+V\left\langle T_{V} V, N\right\rangle-2 \varepsilon_{1} \tau\left\langle T_{V} V, X\right\rangle\right) V
\end{aligned}
$$


Then we conclude that the condition $\Delta H=\lambda H$ is equivalent to the following system of equations:

$$
\left\{\begin{array}{c}
2\left(k X(\alpha)+\varepsilon_{1} \varepsilon_{3} V(\alpha) \tau\right) \\
+\alpha\left(X(k)+\varepsilon_{1} \varepsilon_{3} V(\tau)+\left\langle T_{V} V, X\right\rangle\left(2 \varepsilon_{1} \varepsilon_{2} \varepsilon_{3} \alpha-2 \varepsilon_{3} k\right)\right)=0 \\
-\varepsilon_{1} X^{2}(\alpha)-\varepsilon_{3} V^{2}(\alpha)+\varepsilon_{1} \varepsilon_{3}\left\langle T_{V} V, X\right\rangle X(\alpha) \\
+\varepsilon_{2} \alpha\left(2 k^{2}+2 \varepsilon_{1} \varepsilon_{3} \tau^{2}+4 \alpha^{2}-4 \varepsilon_{1} \varepsilon_{2} \alpha k-\varepsilon_{2} \lambda\right)=0 \\
2 \varepsilon_{1} \varepsilon_{3}(-V(\alpha) k+X(\alpha) \tau) \\
+\alpha\left(6 \varepsilon_{2} \varepsilon_{3} V(\alpha)+\varepsilon_{1} \varepsilon_{3} X(\tau)-2 \varepsilon_{1} \tau\left\langle T_{V} V, X\right\rangle\right)=0
\end{array}\right.
$$

where we have used the formula (2.3) and the fact that the curvature $k$ is constant on the fibres, which implies that $V(k)=0$.

\section{MAin Results}

A particularly interesting case arises when the submersion $\pi$ has totally geodesic fibres, i.e., the tensor $T$ vanishes. Then we have to say that there are enough important well-known submersions that are totally geodesic, such as the Hopf fibration.

In such a case, there exists a simple relation between $\alpha$ and $k$, because the equation (2.3) is reduced to

$$
\alpha=\frac{1}{2} \varepsilon_{1} \varepsilon_{2} k
$$

so $V(\alpha)=0$, and (2.4) can be simplified to

$$
\left\{\begin{array}{l}
3 \varepsilon_{1} k X(k)+\varepsilon_{3} k V(\tau)=0, \\
-\varepsilon_{2} X^{2}(k)+\varepsilon_{1} k\left(k^{2}+2 \varepsilon_{1} \varepsilon_{3} \tau^{2}-\varepsilon_{2} \lambda\right)=0, \\
X(k) \tau+\frac{1}{2} k X(\tau)=0 .
\end{array}\right.
$$

We can already state and prove the following characterization theorem for the total lifts of curves satisfying the condition $\Delta H=\lambda H$, when the submersion $\pi$ is totally geodesic.

Theorem 3.1. Let $\pi: M_{s}^{3} \rightarrow B_{r}^{2}$ be a totally geodesic pseudo-Riemannian submersion and let $\beta: I \rightarrow B_{r}^{2}$ be a non-null regular curve. Then its total lift $P=\pi^{-1}(\beta)$ satisfies the condition $\Delta H=\lambda H$, where $\lambda$ is constant, if and only if the surface $P$ is minimal or the horizontal lifts of $\beta$ are helices with curvature $k$ and torsion $\tau$ related by

$$
\lambda=\varepsilon_{2}\left(k^{2}+2 \varepsilon_{1} \varepsilon_{3} \tau^{2}\right) .
$$

Proof. Suppose that the surface $P$ satisfies the equation $\Delta H=\lambda H$. Since the Lie bracket $[X, V]$ is vertical and $V(k)=0$, it can be concluded that $V(X(k))=0$ and $V(X(X(k)))=0$. So, by differentiating the second equation of (3.1) with respect to $V$, we have

$$
k \tau V(\tau)=0 .
$$

This equation implies that $k V(\tau)=0$, which is applied to the first equation of (3.1) to conclude that the curvature function $k$ is constant. If $k$ is not equal to zero, the second equation of (3.1) yields the relation between $k$ and $\tau$, from which $\tau$ has to be constant.

The converse is reduced to an immediate and direct checking. 
As a consequence, we can obtain that the Chen conjecture is verified in particular cases. Recall that a surface $P \subset M^{3}$ is biharmonic when $P$ has harmonic mean curvature vector field, i.e., $\Delta H=0$.

Corollary 3.2. Let $\pi: M^{3} \rightarrow B^{2}$ be a totally geodesic Riemannian submersion and let $\beta: I \rightarrow B^{2}$ be a regular curve. Then its total lift $P=\pi^{-1}(\beta)$ is biharmonic if and only if it is minimal.

Corollary 3.3. Let $\pi: M_{1}^{3} \rightarrow B_{1}^{2}$ be a totally geodesic pseudo-Riemannian submersion and let $\beta: I \rightarrow B_{1}^{2}$ be a spacelike regular curve. Then its total lift $P=\pi^{-1}(\beta)$ is biharmonic if and only if it is minimal.

Another interesting case arises when the tensor $A$ vanishes, i.e., the submersion has integrable horizontal distribution. For instance, the projection of any warped product over its base space is a submersion of this type. Since the torsion is written as $\tau=\left\langle A_{X} V, N\right\rangle$, in this case it vanishes. Therefore, the equations (2.4) reduce to the system

$$
\left\{\begin{array}{l}
2 k X(\alpha)+\alpha\left(X(k)+\left\langle T_{V} V, X\right\rangle\left(2 \varepsilon_{1} \varepsilon_{2} \varepsilon_{3} \alpha-2 \varepsilon_{3} k\right)\right)=0 \\
-\varepsilon_{1} X^{2}(\alpha)-\varepsilon_{3} V^{2}(\alpha)+\varepsilon_{1} \varepsilon_{3}\left\langle T_{V} V, X\right\rangle X(\alpha) \\
\quad+\varepsilon_{2} \alpha\left(2 k^{2}+4 \alpha^{2}-4 \varepsilon_{1} \varepsilon_{2} \alpha k-\varepsilon_{2} \lambda\right)=0 \\
V(\alpha)\left(-\varepsilon_{1} k+3 \varepsilon_{2} \alpha\right)=0
\end{array}\right.
$$

which yields the following result.

Proposition 3.4. Let $\pi: M_{s}^{3} \rightarrow B_{r}^{2}$ be a pseudo-Riemannian submersion with integrable horizontal distribution and let $\beta: I \rightarrow B_{r}^{2}$ be a non-null regular curve. If $P=\pi^{-1}(\beta)$ satisfies the condition $\Delta H=\lambda H$, where $\lambda$ is constant, then the mean curvature $\alpha$ of $P$ is constant on the fibres.

Proof. We have to show that $V(\alpha)=0$. Otherwise, there is a point $p \in P$ such that $V(\alpha) \neq 0$ and a neighborhood $\mathcal{U}$ of $p$ where $V(\alpha) \neq 0$. Since $V(k)=0$, the third equation of (3.2) implies that $V(\alpha)=0$ in $\mathcal{U}$, which is a contradiction.

In view of the last proposition, we could wonder if every lift $P$ of $\beta$ satisfying the condition $\Delta H=\lambda H$ when $A=0$ has constant mean curvature. The answer is negative and we are going to give a counterexample which is a biharmonic surface with non-constant mean curvature in an open subset of $\mathbb{R}^{3}$ furnished with a non-flat Riemannian metric. In particular, it means that the Chen conjecture is not true for $\mathbb{R}^{3}$ with a non-flat Riemannian metric.

Example 3.5. Let us consider $\mathbb{R}^{1}$ endowed with the Euclidean metric and $B=$ $\left\{(s, t) \in \mathbb{R}^{2}: s>0\right\}$ endowed with the metric tensor

$$
d s \otimes d s+\varphi(s, t)^{2} d t \otimes d t, \quad \varphi(s, t)=\frac{\mu s}{\sqrt{2}\left(1+t^{2}\right)}, \quad \mu \in \mathbb{R} \backslash\{0\} .
$$

Let $M^{3}=B \times_{f} \mathbb{R}$ be the warped product with warping function $f(t)=e^{\mu \arctan t}$. Then the canonical projection $\pi: M^{3} \rightarrow B$ is a Riemannian submersion with integrable horizontal distribution. Moreover, if we consider the curve $\beta:(0, \infty) \rightarrow$ $B$ defined by $\beta(s)=\left(s, t_{0}\right)$, it is easy to see that its total lift satisfies the condition $\Delta H=0$ and it does not have constant mean curvature. 
Constant mean curvature surfaces play a fundamental role in many fields of Differential Geometry and Physics. For this reason, we are going to characterize all lifts via a submersion with constant mean curvature.

Theorem 3.6. Let $\pi: M_{s}^{3} \rightarrow B_{r}^{2}$ be a pseudo-Riemannian submersion with integrable horizontal distribution and let $\beta: I \rightarrow B_{r}^{2}$ be a non-null regular curve. Then its total lift $P=\pi^{-1}(\beta)$ satisfies the condition $\Delta H=\lambda H$ and has constant mean curvature if and only if at least one of the following conditions holds:

(a) $P$ is minimal,

(b) $k$ is constant and $P$ is totally umbilical; in this case $\lambda=2 \varepsilon_{2} k^{2}$,

(c) $k$ is constant and the fibres are geodesics in $P$ having constant curvature $\mu$ in $M_{s}^{3}$; in this case $\lambda=\varepsilon_{2}\left(k^{2}+\mu^{2}\right)$.

Proof. First suppose that $P$ satisfies the condition $\Delta H=\lambda H$ and its mean curvature function is constant and not equal to zero. Then the system (3.2) is reduced to

$$
\left\{\begin{array}{l}
X(k)+\left\langle T_{V} V, X\right\rangle\left(2 \varepsilon_{1} \varepsilon_{2} \varepsilon_{3} \alpha-2 \varepsilon_{3} k\right)=0, \\
2 k^{2}+4 \alpha^{2}-4 \varepsilon_{1} \varepsilon_{2} \alpha k-\varepsilon_{2} \lambda=0 .
\end{array}\right.
$$

From the last equation, we deduce that $k$ is constant. By using the equation (2.3), the above system can be written as

$$
\left\{\begin{array}{l}
\left\langle T_{V} V, X\right\rangle\left(\varepsilon_{1}\left\langle T_{V} V, N\right\rangle-\varepsilon_{3} k\right)=0 \\
k^{2}+\left\langle T_{V} V, N\right\rangle^{2}-\varepsilon_{2} \lambda=0
\end{array}\right.
$$

Then, from the first equation, we get that $\left\langle T_{V} V, X\right\rangle$ is identically zero or $k=$ $\varepsilon_{1} \varepsilon_{3}\left\langle T_{V} V, N\right\rangle$. The last one together with the relation given by the second equation implies the assertions of the proposition.

Conversely, if $P$ is minimal, then $P$ trivially satisfies the equation $\Delta H=\lambda H$. If (b) or (c) holds, then the mean curvature $\alpha$ of $P$ is constant. In this case, the equation $\Delta H=\lambda H$ is equivalent to the system of equations (3.3), which is trivially satisfied.

We can get the following consequence for biharmonic lifts.

Corollary 3.7. Let $\pi: M_{s}^{3} \rightarrow B_{r}^{2}$ be a pseudo-Riemannian submersion with integrable horizontal distribution and let $\beta: I \rightarrow B_{r}^{2}$ be a non-null regular curve. Then its total lift $P$ is a biharmonic surface of constant mean curvature if and only if $P$ is minimal.

Finally, Theorem 3.6 allows us to find examples of lifts with constant mean curvature satisfying $\Delta H=\lambda H$.

Example 3.8. Let $g: \mathbb{R} \rightarrow \mathbb{R}$ be a positive differentiable function and let $f: \mathbb{R}^{2} \rightarrow$ $\mathbb{R}$ be the function given by $f(x, y)=g\left(x^{2}+y^{2}\right)$. Let us consider the warped product $\mathbb{R}^{2} \times{ }_{f} \mathbb{R}_{s}^{1}, s=0,1$, where $\mathbb{R}^{2}$ and $\mathbb{R}_{s}^{1}$ are equipped with the canonical metrics. Then the right cylinders $\mathfrak{C} \times \mathbb{R}$ over a circle $\mathfrak{C} \subset \mathbb{R}^{2}$ centered at the origin have constant mean curvature and satisfy the condition $\Delta H=\lambda H$. An example of type (b) in Theorem 3.6 that is not of type (c) is obtained by putting $f(x, y)=\sqrt{x y}, x y>0$ and taking a unit circle. 


\section{REFERENCES}

1. L. J. Alías, A. Ferrández and P. Lucas, Classifying pseudo-Riemannian hypersurfaces by means of certain characteristic differential equations, The problem of Plateau, World Sci. Publishing, River Edge, NJ, 1992, pp. 53-75. MR 94h:53087

2. M. Barros, A. Ferrández and P. Lucas, Lawson-type problems in nonstandard 3-spheres, Quart. J. Math. Oxford, 50 (1999), 385-388. MR 2000j:53078

3. M. Barros, A. Ferrández, P. Lucas and M. A. Meroño, Hopf cylinders, B-scrolls and solitons of the Betchov-Da Rios equation in the three-dimensional anti-de Sitter space, C. R. Acad. Sci. Paris Sér. I Math., 321 (1995), 505-509. MR 97h:53009

4. M. Barros and O. J. Garay, On submanifolds with harmonic mean curvature, Proc. Amer. Math. Soc., 123 (1995), 2545-2549. MR 95j:53082

5. B. Y. Chen, Finite-type pseudo-Riemannian submanifolds, Tamkang J. of Math., 17 (1986), 137-151. MR 88h:53059

6. B. Y. Chen, Some open problems and conjectures on submanifolds of finite type, Soochow J. Math., 17 (1991), 169-188. MR 92m:53091

7. B. Y. Chen and S. Ishikawa, Biharmonic surfaces in pseudo-Euclidean spaces, Memoirs Fac. Sci. Kyushu Univ. Ser. A Math, 45 (1991), 323-347. MR 92k:53113

8. A. Ferrández, P. Lucas and M. A. Meroño, Biharmonic Hopf cylinders, Rocky Mountain J. Math., 28 (1998), 957-975. MR 99k:53126

9. J.-H. Kwon y Y. J. Suh, On sectional and Ricci curvatures of semi-Riemannian submersions, Kodai Math. J., 20 (1997), 53-66. MR 98b:53062

10. B. O'Neill, The fundamental equations of a submersion, Michigan Math. J., 13 (1966), 459469. MR 34:751

11. U. Pinkall, Hopf tori in $\mathbb{S}^{3}$, Invent. Math., 81 (1985), 379-386. MR 86k:53075

Departamento de Matemáticas, Universidad de Murcia, 30100 Espinardo, Murcia, SPAIN

E-mail address: majava@um.es

Departamento de Matemáticas, Universidad de Murcia, 30100 Espinardo, Murcia, SPAIN

E-mail address: mamb@um.es 